\title{
Artigo/Article
}

\section{Características clínico-evolutivas de 134 pacientes com histoplasmose disseminada associada a SIDA no Estado do Ceará}

\author{
Clinical and evolutionary characteristics of 134 patients with disseminated histoplasmosis \\ associated with AIDS in the State of Ceará
}

Lícia Borges Pontes ${ }^{1}$, Terezinha do Menino Jesus Silva Leitão ${ }^{2}$, Gabrielle Gurgel Lima ${ }^{3}$, Emília Santiago Gerhard ${ }^{3}$ e Thyago Araújo Fernandes ${ }^{3}$

\begin{abstract}
RESUMO
Introdução: Desde o início da epidemia de HIV no Ceará, histoplasmose disseminada tem sido detectada com frequência em pacientes com aids. Métodos: De modo a conhecer características clínico-laboratoriais, evolução e sobrevida da co-infecção HD/AIDS, analisou-se retrospectivamente 134 prontuários casos de HD internados de 1999 a 2005 no hospital referência para HIV no Ceará. Resultados: Pacientes com HD apresentaram maior frequência de febre diária, tosse, perda de peso, hepatoesplenomegalia e insuficiência renal aguda. Diagnóstico foi dado por pesquisa e/ou cultura. À admissão, foram fatores de risco relacionados ao óbito de pacientes com HD: vômitos, dispnéia, insuficiência respiratória e IRA, hemoglobina $\leq 8 \mathrm{~g} / \mathrm{L}$, uréia $\geq 40 \mathrm{mg} / \mathrm{dL}$ e creatinina $\geq 1,5 \mathrm{mg} / \mathrm{dL}$. Conclusões: Pacientes com HD apresentaram caracteristicamente febre mais elevada, internamentos anteriores por infecção respiratória, mais complicações clínicas e como fatores independentes para óbito, anemia importante e elevação de uréia.
\end{abstract}

Palavras-chaves: Histoplasmose disseminada. AIDS. Ceará. Doença oportunista.

\begin{abstract}
Introduction: Since the beginning of the HIV epidemic in Ceará, disseminated histoplasmosis (DH) has often been detected among AIDS patients. Methods: In order to investigate the clinical and laboratory characteristics, evolution and survival of cases of DH/AIDS coinfection, the medical records on 134 cases of DH admitted to a reference hospital in Ceará between 1999 and 2005 were analyzed. Results: Patients with DH presented higher frequency of daily fever, coughing, weight loss, enlarged liver and spleen and acute kidney failure. The diagnosis was made using investigation and/or cultures. At admission, the following were risk factors for death among DH patients: vomiting, dyspnea, respiratory failure, acute kidney failure, hemoglobin $\leq 8 \mathrm{~g} / \mathrm{l}$, urea $\geq 40 \mathrm{mg} / \mathrm{dl}$ and creatinine $\geq 1.5 \mathrm{mg} / \mathrm{dl}$. Conclusions: Patients with DH characteristically presented higher fever, previous hospitalization due to respiratory infection and more clinical complications. Significant anemia and elevated urea were independent risk factors for death among $\mathrm{DH}$ patients.
\end{abstract}

Key-words: Disseminated histoplasmosis. AIDS. Ceará. Opportunistic disease.

1. Departamento de Infectologia, Hospital São José de Doenças Infecciosas, Universidade Federal do Ceará. Fortaleza, CE. 2. Universidade Federal de São Paulo, São Paulo, SP. 3. Departamento de Medicina, Universidade Federal do Ceará. Fortaleza, CE

Endereço para correspondência: Dra Gabrielle Gurgel Lima. UFCE. Rua Dr Gilberto Studart 1493/1701 Papicu, 60190-750 Fortaleza, CE.

Tel: 5585 3234-2636

e-mail: gabi_gurgel@yahoo.com.br

Recebido para publicação em 29/10/2009

Aceito em 11/01/2010

\section{INTRODUÇÃO}

A histoplasmose disseminada (HD) é uma infecção oportunista definidora de AIDS em $22-85 \%$ dos pacientes de áreas endêmicas ${ }^{1}$. Em 1994, o Centers for Disease Control and Prevention (CDC) definiu como casos graves de HD aqueles com um ou mais dos seguintes critérios: temperatura maior ou igual a $39^{\circ} \mathrm{C}$, pressão arterial sistólica $<90 \mathrm{mmHg}, \mathrm{pO}_{2}<70 \mathrm{mmHg}$, perda de peso > 5\% do peso corporal total, escore de Karnofsky $<70$, hemoglobina $<10 \mathrm{~g} / \mathrm{dL}$, contagem de neutrófilos $<1000$ células $/ \mathrm{mm}^{3}$, contagem de plaquetas $<100.000$ células $/ \mathrm{mm}^{3}$, aspartato aminotransferase $($ AST $)>2,5$ vezes o normal, bilirrubina ou creatinina $>$ duas vezes o normal, albumina $<3,5 \mathrm{~g} / \mathrm{dL}$, coagulopatia, disfunção de outro órgão ou meningite associada $^{2}$.

Ahistoplasmose apresenta incidência desconhecida no Brasil. Segundo dados da coordenação municipal de Doença Sexualmente Transmissível/AIDS de Fortaleza, HD tem sido nos anos recentes a segunda infecção sistêmica mais notificada em pacientes com AIDS. De 1983 a 2006, foram notificados 6.466 casos de AIDS em Fortaleza e 182 casos de histoplasmose disseminada ${ }^{3}$.

Estudando os fatores de risco para morte de pacientes de hospitais referência do Ceará, em 2006, Daher cols encontraram que, para análise univariada, diarréia, manifestações neurológicas, hemoglobina $<8,0 \mathrm{~g} / \mathrm{dL}$, uréia $>2$ vezes o limite superior de normalidade (LSN), creatinina $>1,5 \mathrm{mg} / \mathrm{dL}$ e AST $>2,5$ vezes $\mathrm{LSN}$ foram fatores associados a um aumento no risco de morte ${ }^{4}$. Na análise multivariada, baixos níveis de hemoglobina, níveis elevados de AST, insuficiência renal aguda e insuficiência respiratória foram fatores de risco independentes para morte em pacientes com AIDS e $\mathrm{HD}^{4}$. Este foi o primeiro estudo de fatores de risco para morte realizado no Brasil.

Esse estudo se propõe a conhecer como os pacientes evoluem ao longo dos anos e determinar 
qual período é crítico para sua abordagem clínica, no intuito de reduzir a elevada mortalidade ainda associada a essa patologia. Através de acompanhamento clínico pretendemos analisar a ainda o uso de profilaxia secundária, recaídas de HD e óbito.

\section{MÉTODOS}

Este estudo analisou retrospectivamente os prontuários médicos de pacientes com AIDS com diagnóstico de HD internados no Hospital São José de Doenças Infecciosas do Ceará entre os anos de 1999 e 2005.

Foram estudados pacientes com AIDS segundo definição do Ministério da Saúde do Brasil ${ }^{2}$, com idade mínima de 18 anos, de ambos os sexos, que realizaram pesquisa e/ou cultura para fungo de creme leucocitário, medula óssea, sangue ou tecidos sólidos duranteinternamento no HSJ no período de janeiro de 1999 a dezembro de 2005.

Os casos de HD foram definidos como pacientes com AIDS internados no HSJ com o primeiro episódio de HD no período do estudo, confirmados por pesquisa direta e/ou culturas de fungo positivas por Histoplasma capsulatum.

Foram pesquisados os arquivos do laboratório do HSJ e registros do laboratório central (LACEN) para identificação de pacientes nos quais foram realizadas pesquisa direta e ou cultura de material biológico para fungo. Os prontuários foram consultados no SAME (Serviço de Arquivos Médicos e Estatística) e uma ficha semi-estruturada preenchida contendo dados sociodemográficos, clínicos, laboratoriais e evolutivos dos pacientes. As informações foram obtidas até dois anos do internamento e a coleta de dados foi encerrada em maio de 2008, sempre levando em consideração a data do comparecimento à última consulta.

Um banco de dados foi construído com as variáveis coletadas através do questionário. Os programas Excel e Statistical Package for Social Sciences (SPSS Inc. Chicago, IL) foram utilizados para todas as análises paramétricas e não-paramétricas. Para a análise de homogeneidade e de associação em tabelas de contingência, foi utilizado o teste exato de Fisher. O Teste de Kolmogorov-Smirnov foi usado para testar a normalidade na distribuição das variáveis contínuas. Quando à distribuição, não era significativamente diferente da distribuição normal, foi usado o teste T-Student e no caso contrário foi utilizado o teste de Mann Whitney.

Para a análise de fatores de risco no contexto multidimensional, foi usada a regressão logística. Para estimar a sobrevida dos pacientes foi utilizado o teste de Kaplan-Meier. Os resultados foram considerados significantes para um valor de $p<0,05$. Razão de prevalência (odds ratio) e intervalo de confiança de $95 \%$ também foram calculados.

\section{Ética}

O projeto foi aprovado pelo Comitê de ética em Pesquisa do HSJ, segundo os requisitos da resolução CNS 196/96 e suas complementares que regulamentam pesquisa em seres humanos.

\section{RESULTADOS}

No período de 1999 a 2005, foram diagnosticados 191 casos de HD e AIDS no HSJ. Destes, 134 foram incluídos no estudo. A média de idade foi de 35,8 anos (desvio padrão $=9,781$ ). O critério de definição de AIDS mais frequente nos pacientes foi CDC modificado $(61,9 \%)$, seguido do Caracas $(29,9 \%)$. Em relação ao tempo entre a sorologia para HIV e o diagnóstico de AIDS, a maioria dos doentes tinham menos que 30 dias de intervalo.

Cinquenta e dois $(40,3 \%)$ pacientes tiveram internamentos anteriores por HIV ( $p=0,254)$; destes, $48 \%$ tiveram até 60 dias entre a internação anterior e a atual. Vinte e seis (50\%) pacientes tiveram internamento anterior por infecção respiratória não tuberculosa.

A maioria dos pacientes teve início dos sintomas até trinta dias antes do internamento. Febre, tosse, perda de peso, diarréia, vômitos, hepatomegalia, esplenomegalia, presença de lesões de pele sugestivas de histoplasmose, icterícia e insuficiência renal aguda foram condições, à admissão, significativamente mais presentes.

Na Tabela 1, estão os principais exames laboratoriais dos pacientes à admissão, separados por ponto de corte. Observa-se que a análise de prevalência mostrou diferença para níveis de hemoglobina

TABELA 1 - Exames laboratoriais à admissão de pacientes com HD separados por ponto de corte (HSJ, 1999-2005).

\begin{tabular}{|c|c|c|c|}
\hline Achados laboratoriais & HD & $\%$ & OR (IC 95\%) \\
\hline Hemoglobina $\mathrm{g} / \mathrm{L}$ & & & $1,963(1,133-3,402)$ \\
\hline$\leq 8$ & 50 & 40,7 & \\
\hline$>8$ & 73 & 59,3 & \\
\hline Leucócitos céls $/ \mathrm{mm}^{3}$ & & & $2,410(1,432-4,058)$ \\
\hline$<3.500$ & 68 & 52,7 & \\
\hline$\geq 3.500$ & 61 & 47,3 & \\
\hline Plaquetas céls $/ \mathrm{mm}^{3}$ & & & $6,413(3,478-11,824)$ \\
\hline$\leq 100.000$ & 66 & 55,9 & \\
\hline$>100.000$ & 52 & 44,1 & \\
\hline Creatinina $\mathrm{mg} / \mathrm{dL}$ & & & $1,992(1,030-3,855)$ \\
\hline$\geq 1,5$ & 34 & 26,2 & \\
\hline$<1,5$ & 96 & 73,8 & \\
\hline Uréia mg/dL & & & $2,506(1,448-4,337)$ \\
\hline$\geq 40$ & 64 & 50,4 & \\
\hline$<40$ & 63 & 49,6 & \\
\hline LDH UI/L & & & $15,019(7,458-30,247)$ \\
\hline$\geq 2 \mathrm{xLSN}$ & 83 & 74,8 & \\
\hline$<2 x L S N$ & 28 & 25,2 & \\
\hline AST UI/L & & & $8,375(3,863-18,155)$ \\
\hline$\geq 3 \mathrm{xLSN}$ & 55 & 55,6 & \\
\hline$<3 x L S N$ & 44 & 44,4 & \\
\hline ALT UI/L & & & $5,858(1,665-20,612)$ \\
\hline$\geq 3 \mathrm{xLSN}$ & 19 & 19,2 & \\
\hline$<3 x L S N$ & 80 & 80,8 & \\
\hline TAP $\%$ & & & $2,715(1,185-6,223)$ \\
\hline$<70$ & 32 & 68,1 & \\
\hline$\geq 70$ & 15 & 31,9 & \\
\hline FA UI/L & & & $5,958(1,793-19,805)$ \\
\hline$\geq 300$ & 22 & 47,8 & \\
\hline$<300$ & 24 & 52,2 & \\
\hline Albumina, $\mathrm{g} / \mathrm{dL}$ & & & -- \\
\hline$<3,0$ & 26 & 59,1 & \\
\hline$\geq 3,0$ & 18 & 40,9 & \\
\hline $\mathrm{CD} 4+$ & & & $2,15(1,119-4,13)$ \\
\hline$\leq 100$ & 61 & 74,4 & \\
\hline$>100$ & 21 & 25,6 & \\
\hline
\end{tabular}

LDH: lactato desidrogenase, AST: aspartato aminotransferase, ALT: alanina aminotransferase, TAP: tempo de ativação da protrombina, FA: fosfatase alcalina, LSN: limite superior da normalidade. HD: histoplasmose disseminada, OR: odds ratio. 
menores ou iguais a oito. Nota-se que a maioria dos doentes apresenta valores de $\mathrm{CD} 4 \leq 100 \mathrm{cel} / \mathrm{mm}^{3}$.

Cento e onze pacientes realizaram estudo radiográfico do tórax. Destes, 80 apresentaram alguma anormalidade. Infiltrado intersticial foi a alteração mais presente (45\%), seguida de infiltrado difuso (17,1\%) e misto (13,3\%). Derrame pleural também foi um achado presente.

O diagnóstico de $\mathrm{HD}$ por cultura foi feito em 66 pacientes e por biópsia em dois.

Insuficiência renal aguda, insuficiência respiratória e sepse foram as complicações mais frequentes $(p<0,05)$. Desfecho para óbito durante o internamento foi de 32,8\% (Tabela 2). Dos pacientes que tiveram infecção respiratória não tuberculosa, em internação anterior, (33), 42,2\% destes evoluíram para óbito na internação seguinte.

Foram fatores de risco relacionados ao óbito à admissão hospitalar: vômitos, dispnéia e dor pleurítica; dentre as complicações destacaramse insuficiência respiratória e renal aguda, e dos exames laboratoriais a hemoglobina, uréia, creatinina, AST eLDH. Outros fatores de risco para óbito foram: admissão em unidade de terapia intensiva, sepse, médias altas de AST, uréia e creatinina e média baixa de hemoglobina à admissão e médias altas de LDH, uréia e creatinina à evolução (Tabela 3).

Foram testadas as variáveis com um nível de significância de $15 \%(p<0,15)$ para o óbito num modelo de análise multivariada. Ao nível de significância de $10 \%$, acurácia de $78 \%$, sensibilidade de $70,8 \%$ e especificidade de $81 \%$; foram fatores de risco independente para o óbito: hemoglobina $<8 \mathrm{mg} / \mathrm{dL}(p=0,0360)$ e uréia $\geq 40 \mathrm{mg} / \mathrm{dL}$ $(p=0,0096)$ à admissão.

TABELA 2 - Eventos clínicos durante o internamento de pacientes (HSJ, 1999-2005).

\begin{tabular}{|c|c|c|c|}
\hline \multirow[b]{2}{*}{ Eventos clínicos* } & \multicolumn{2}{|c|}{$\mathrm{HD}\left(\mathrm{n}^{\circ}=134\right)$} & \multirow[t]{2}{*}{$\mathrm{p}$} \\
\hline & $\mathrm{n}^{\mathrm{o}}$ & $\%$ & \\
\hline Insuficiência respiratória aguda & 70 & 56 & 0,000 \\
\hline Insuficiência respiratória & 52 & 38,8 & 0,046 \\
\hline Sepse & 29 & 21,6 & 0,044 \\
\hline Internamento em UTI & 29 & 21,8 & 0,527 \\
\hline Óbito & 44 & 32,8 & 0,213 \\
\hline
\end{tabular}

${ }^{*}$ Desenvolvida durante a evolução, HD: histoplasmose disseminada.

TABELA 3 - Achados clínicos e laboratoriais na admissão hospitalar e óbito em pacientes com HD (HSJ, 1999-2005).

\begin{tabular}{|c|c|c|c|c|c|c|c|}
\hline & \multicolumn{5}{|c|}{ Histoplasmose disseminada } & \multirow[b]{3}{*}{ OR } & \multirow[b]{3}{*}{ IC 95\% } \\
\hline & \multicolumn{2}{|c|}{ óbito $(\mathrm{n}=44)$} & \multicolumn{3}{|c|}{ não óbito $(\mathrm{n}=90)$} & & \\
\hline & $\mathrm{n}^{\mathrm{o}}$ & $\%$ & $\mathrm{n}^{\mathrm{o}}$ & $\%$ & $\mathrm{p}$ & & \\
\hline \multicolumn{8}{|l|}{ Sintomas } \\
\hline vômitos & 27 & 61,4 & 35 & 39,8 & 0,026 & 2,405 & $1,145-5,051$ \\
\hline dispnéia & 25 & 58,1 & 29 & 32,2 & 0,008 & 2,921 & $1,380-6,185$ \\
\hline dor pleurítica & 9 & 20,5 & 7 & 8 & 0,049 & 2,976 & $1,026-8,626$ \\
\hline sangramento & 10 & 23,3 & 9 & 10,3 & 0,065 & --- & --- \\
\hline \multicolumn{8}{|l|}{ Complicações } \\
\hline IRA admissão & 25 & 56,8 & 29 & 32,2 & 0,009 & 2,768 & $1,317-5,815$ \\
\hline Insuf. resp. adm. & . 14 & 31,8 & 1 & 1,1 & 0,000 & 41,533 & $5,238-329,316$ \\
\hline \multicolumn{8}{|l|}{ Laboratório } \\
\hline hemoglobina $\leq 8$ & 22 & 57,9 & 28 & 32,9 & 0,011 & 2,799 & $1,274-6,149$ \\
\hline uréia $\geq 40$ & 30 & 73,2 & 34 & 39,5 & 0,001 & 4,171 & $1,846-9,423$ \\
\hline creatinina $\geq 1,5$ & 18 & 43,9 & 16 & 18 & 0,003 & 3,571 & $1,572-8,111$ \\
\hline AST $>114$ & 5 & 45,5 & 5 & 11,1 & 0,018 & 6,667 & $1,476-30,106$ \\
\hline $\mathrm{LDH}>920$ & 8 & 61,5 & 6 & 15,8 & 0,003 & 8,533 & $2,068-35,209$ \\
\hline
\end{tabular}

LDH: lactato desidrogenase, AST: aspartato aminotransferase, IC: intervalo de confiança, HD: histoplasmose disseminada, OR: odds ratio. IRA: Insuficiência respiratória aguda.
A maioria dos pacientes foi tratada com anfotericina B (87,5\%), com itraconazol $(8,6 \%)$ e com fluconazol (2,3\%). A maioria que recebeu alta hospitalar fez profilaxia secundária para HD (81\%), com itraconazol oral $(79,4 \%)$ e de forma regular $(61,2 \%)$. Foi notada recaída por $\mathrm{HD}$ em $21,8 \%$ dos casos, durante todo período do estudo.

Durante o acompanhamento de dois anos, $52,2 \%$ dos pacientes foram a óbito. Dos que morreram no primeiro mês (41) o tempo médio de vida foi 0,291 mês ( 8,73 dias), com $\mathrm{DP}=0,235$ (mínimo 0 e máximo 0,98 mês).

\section{DISCUSSÃO}

Na última década, a incidência da histoplasmose mais que duplicou entre os pacientes com AIDS no Ceará (Secretaria Municipal de Saúde). Foram 191 casos de HD e AIDS, além de 4.529 internamentos por AIDS de janeiro de 1999 a dezembro de 2005. Estudo retrospectivo realizado no Panamá, de janeiro de 1997 a dezembro de 2003, revelou 2379 internamentos por AIDS no período, e 184 tinham $\mathrm{HD}^{1}$.

A maioria (61,9\%) dos pacientes com $\mathrm{HD}$ teve seu diagnóstico de AIDS pelo critério CDC, o que denota um estado de imunossupressão muito avançado. HD é a primeira infecção oportunista em $22-85 \%$ dos pacientes com AIDS $^{5-7,15}$. Aproximadamente, $70 \%$ dos pacientes tiveram diagnóstico de HIV e AIDS quase que simultaneamente, com menos de 30 dias de intervalo entre a realização e confirmação da sorologia e o diagnóstico de AIDS.

Mais de um terço (38\%) dos pacientes afirmou fazer uso de TARV no mês que precedeu o diagnóstico, mas somente $41,1 \%$ destes afirmaram fazer uso regular da medicação, mostrando um perfil de má adesão a TARV. No Panamá, de 1997 a 2003, dos 104 pacientes estudados, apenas 14 (13,5\%) estavam em uso de terapia antiretroviral de alta potência (HAART) no momento do diagnóstico de $\mathrm{HD}^{1}$.

Cinquenta e dois $(40,3 \%)$ pacientes já tiveram internamento anterior por AIDS. Destes, 25 tiveram menos de 60 dias entre o internamento anterior e o do diagnóstico de HD, e 26 tiveram diagnóstico de infecção pulmonar não tuberculosa (pneumonia por $P$. jiroveci ou pneumonia adquirida na comunidade) neste internamento anterior. Isso sugere fortemente que esses pacientes podiam já ter HD e foram tratados parcialmente com sulfametoxazoltrimetoprim ou com antifúngicos sistêmicos para alguma micose associada ${ }^{8}$. Dos pacientes que tiveram infecção respiratória não tuberculosa em internação anterior, $42,4 \%$ foram a óbito na internação seguinte $(\mathrm{p}=0,043)$. Isso comprova como o atraso diagnóstico piora o prognóstico da doença.

Análise bivariada mostrou que os sinais e sintomas mais presentes à admissão foram febre, tosse, perda de peso, diarréia, vômitos, hepatoesplenomegalia, lesões de pele sugestivas de $\mathrm{HD}$, insuficiência renal aguda e icterícia $(p<0,05)$. Estudo de 104 pacientes no Panamá evidenciou febre, sintomas respiratórios, perda de peso, diarréia, hepatomegalia e/ou esplenomegalia e lesões de pele ${ }^{1}$.

Pacientes com HD tiveram médias mais baixas de leucócitos, plaquetas e contagem de células T CD4 $(p<0,05)$, tiveram também médias mais baixas de hematócrito e hemoglobina. Tiveram médias mais altas de creatinina, uréia, LDH, AST, ALT, fosfatase alcalina e CD4 $(p<0,05)$. Daher cols estudaram população semelhante em 
dois hospitais de referência do Ceará, de janeiro de 1995 a janeiro de 2004, e encontraram maiores níveis de creatinina, uréia, LDH, AST, ALT, fosfatase alcalina, bilirrubinas direta e indireta; e níveis mais baixos de hematócrito, hemoglobina, plaquetas, proteínas totais e albumina entre os pacientes com $\mathrm{HD}^{9}$. Houve discordância entre hematócrito, hemoglobina, bilirrubinas, proteínas totais e albumina, que no presente estudo não tiveram suas médias com diferença estatística significante.

Análise bivariada mostrou que pacientes com HD apresentaram com mais frequência níveis de hemoglobina menor ou igual a $8 \mathrm{mg} / \mathrm{dL}$ $(p=0,02)$, de leucócitos menor que 3.500 células $/ \mathrm{mm}^{3}(p=0,001)$, de plaquetas menor ou igual a 100.000 células $/ \mathrm{mm}^{3}(p=0,000)$, de creatinina maior ou igual a $(p=0,049)$, de uréia maior ou igual a $40 \mathrm{mg} /$ $\mathrm{dL}(p=0,001)$, de LDH maior ou igual a duas vezes o LSN $(p=0,000)$, de AST maior ou igual a três vezes o limite superior da normalidade $(p=0,000)$, de ALT maior ou igual a três vezes o LSN $(p=0,002)$, de tempo de protrombina ativado menor que $70 \%(p=0,024)$ e de fosfatase alcalina maior ou igual a 300UI/L $(p=0,003)$. Estudo de 104 pacientes no Panamá evidenciou AST e ou ALT maior que três vezes o LSN (50\%), LDH maior que três vezes o LSN (77\%) e pancitopenia $(36 \%)^{1}$. Em nosso estudo, $74,4 \%$ dos pacientes com HD tiveram CD4 menor que 100 células $/ \mathrm{mm}^{3}$. Contagem de células $\mathrm{T}$ CD4 é reconhecidamente menor em pacientes com $\mathrm{HD}$ do que naqueles com outras doenças oportunistas ${ }^{1,9,10}$.

Dezessete $(21,8 \%)$ pacientes apresentaram recaída da doença em dois anos de acompanhamento. McKinsey cols, em 1999, publicou estudo randomizado duplo-cego comparando o uso de itraconazol como terapia de manutenção versus placebo, e encontrou que 19,5\% dos pacientes em uso de itraconazol e $28,8 \%$ dos pacientes com uso de placebo apresentaram recidivas $(p=0,04)^{11}$. Em 2007, McKinsey cols publicaram que para a $\mathrm{HD}$ o tratamento seria com anfotericina B liposomal por 2 semanas, seguido por itraconazol. A profilaxia seria com itraconazol (200mg por dia) nos pacientes com infecção pelo HIV com células CD4 < 150 células $/ \mathrm{mm}^{3}$ em áreas específicas de endemicidade ${ }^{16}$.

Dez a $20 \%$ dos pacientes com HD e aids evoluem para sepse pelo próprio histoplasma. No presente estudo, 21,6\% dos pacientes desenvolveram sepse. Em estudo retrospectivo no Ceará, de janeiro de 1995 a janeiro de 2004, Daher cols encontraram 9,7\% de sepse. Outras complicações analisadas foram: insuficiência respiratória e IRA ${ }^{9}$. A IRA foi analisada em dois momentos, na admissão (40,3\%) e evolução (56\%). Havia uma presença maior de diarréia e vômitos à admissão, entre os pacientes com $\mathrm{HD}$, o que poderia justificar IRA neste momento.

Neste trabalho, 44 pacientes foram a óbito precocemente, ainda no internamento, enquanto a mortalidade para todo o período do estudo foi de $64(47,8 \%)$ casos. Em estudo retrospectivo de 25 anos na Guiana Francesa, a morte precoce (até 30 dias do início do tratamento) ocorreu em $17,5 \%(31 / 177)$ dos casos; pelo menos $30,7 \%(58 / 189)$ morreram em até seis meses após o início do tratamento ${ }^{10}$.

No presente estudo, foram incluídas num modelo de regressão logística multivariada todas as variáveis que à análise bivariada se mostraram significantes para desfecho óbito. Este trabalho mostrou com $78 \%$ de acurácia, $70,8 \%$ de sensibilidade e $81 \%$ de especificidade, a hemoglobina menor $8 \mathrm{mg} / \mathrm{dL}$ e uréia $\geq 40 \mathrm{mg} / \mathrm{dL}$ à admissão como fatores de risco independentes para morte. Daher cols encontraram como fatores de risco independentes para óbito em pacientes com
HD e AIDS hemoglobina $<8 \mathrm{~g} / \mathrm{dL}, \mathrm{AST}>2,5$ vezes $\mathrm{LSN}$, IRA e insuficiência respiratória ${ }^{12}$. Couppié cols encontraram dispnéia, plaquetas $<100.000 / \mathrm{mm}^{3}$ e $\mathrm{LDH}>2$ vezes $\mathrm{LSN}$ como fatores independentes relacionados a morte em pacientes com HD e AIDS em seu estudo ${ }^{13}$. Wheat colsencontraram que um valor de creatinina> $2,1 \mathrm{mg} / \mathrm{dL}$ e albumina $<3,5 \mathrm{~g} / \mathrm{dL}$ foram associados com um risco aumentado de doença grave ${ }^{14}$.

Concluindo, os pacientes com HD apresentaram caracteristicamente febre mais elevada, internamentos anteriores por infecção respiratória não tuberculosa mais frequentes, mais complicações clínicas, tendo como fatores independentes para óbito hemoglobina menor ou igual a oito e elevação de uréia, além de terem a sua mortalidade significativamente maior durante o internamento por $\mathrm{HD}$. Os pacientes com HD foram diagnosticados em fase avançada da infecção por HIV e, com frequência, através do adoecimento pela histoplasmose. Além disso, apresentaram recaída de $22 \%$.

\section{CONFLITO DE INTERESSE}

Os autores declaram não haver nenhum tipo de conflito de interesse.

\section{SUPORTE FINANCEIRO}

Coordenação de Aperfeiçoamento de Pessoal de Nível Superior (CAPES).

\section{REFERÊNCIAS}

1. Gutierrez ME, Canton A, Sosa N, Puga E, Talavera L. Disseminated histoplasmosis in patients with AIDS in Panama: a review of 104 cases. Clin Infect Dis 2005; 40:1199-1202.

2. Centers for Disease Control and Prevention. Treating Opportunistic Infections Among HIV-Infected Adults and Adolescents: recommendations from CDC, the National Institutes of Health, and the HIV Medicine Association/Infectious Disease Society of America. Morbity and Mortality Weekly Report 2004; 53[RR 15], 34-35.

3. Holanda EM, Mesquita JRL, Pinheiro MZ, Ângelo MRF, Silva TMJ. Importância do creme leucocitário no diagnóstico da Histoplasmose Disseminada em pacientes com AIDS no Hospital São José (HSJ) - Ceará. Rev Soc Bras Med Trop 1998; 31(supl I): 37-37.

4. Daher EF, Barros FAS, Silva Jr GB, Takeda CFV, Mota RMS, Ferreira MT, et al. Risk Factors for Death in acquired immunodeficiency syndrome-associated Disseminated Histoplasmosis. Amer J Trop Med Hyg 2006; 74:600-603.

5. Borges AS, Ferreira MS, Silvestre MT, Nishioka SA, Rocha A. Histoplasmosis in immunodepressed patients: study of 18 cases seen in Uberlandia, MG. Rev Soc Bras Med Trop 1997; 30:119-124.

6. Pietrobon D, Negro-Marquínez L, Kilstein J, Galíndez J, Greca A, Battagliotti C. Disseminated histoplasmosis and AIDS in an Argentine hospital: clinical manifestations, diagnosis and treatment. Enferm Infec Microbiol Clín 2004; 22:156-159.

7. McKinsey DS, Spiegel RA, Hutwagner L, Stanford J, Driks MR, Brewer J, et al. Prospective study of histoplasmosis in patients infected with human immunodeficiency virus: incidence, risk factors, and pathophysiology. Clin Infect Dis 1997; 24:1195-1203.

8. Hajjeh RA, Pappas PG, Henderson H, Lancaster D, Bamberger DM, Skahan KJ, et al. Multicenter case-control study of risk factors for histoplasmosis in human immunodeficiency virus-infected persons. Clin Infect Dis, 2001, 32:12151220 .

9. Daher EF, Silva Jr GB, Barros FA, Takeda CF, Mota RM, Ferreira MT, et al. Clinical and Laboratory features of disseminated histoplasmosis in HIV patients from Brazil. Trop Med Iternat Health 2007; 12:1108-1115. 
10. Huber F, Nacher M, Aznar C, Pierre-Demar M, El Guedj M, Vaz T, et al. AIDSrelated Histoplasma capsulatum var. Capsulatum infection: 25 years experience of French Guiana. AIDS 2008; 22:1047-1053.

11. McKinsey DS, Wheat LJ, Cloud GA, Pierce M, Black JR, Bamberger DM, et al, National Institute of Allergy and Infectious Diseases Mycoses Study Group. Itraconazole prophylaxis for fungal infections in patients with advanced human immunodeficiency virus infection: randomized, placebo-controlled, double-blind study. Clin Infect Dis 1999, 28:1049-1056.

12. Wheat LJ, Kauffman CA. Histoplasmosis. Infect Dis Clin of North America, 2003, 17:1-19.

13. Couppié P, Sobesky M, Aznar C, Bichat S, Clyti E, Bissuel F, et al. Histoplasmosis and acquired immunodeficiency syndrome: a study of prognostic factors. Clin Infect Dis 2004; 38:134-138.

14. Wheat LJ, Chetchotisakd P, Williams B, Connolly P, Shutt K, Hajjeh R. Factors associated with severe manifestations of histoplasmosis in AIDS. Clin Infect Dis 2000; 30: 877-881.

15. Centers for Disease Control and Prevention. 1993 Revised classification system for HIV infection and expanded surveillance case definition for AIDS among adolescents and adults. Morb Mort Week Rep 1993; 41 [RR 17], 1-19.

16. Wheat LJ, Freifeld AG, Kleiman MB, Baddley JW, McKinsey DS, Loyd JE, et al Clinical practice guidelines for the management of patiens with histoplasmosis: 2007 update by the infectious diseases society of america. Clin Infect Dis 2007; $45: 807-825$ 